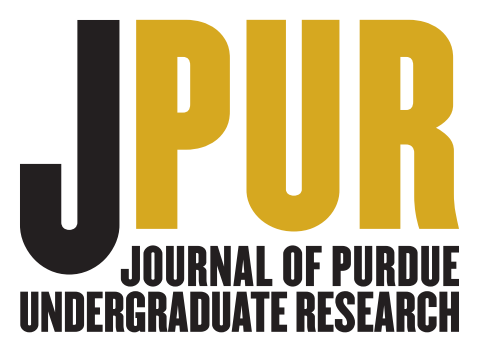

\title{
EDUCATION
}

\section{Study Abroad Journeys: Connecting Participants' Community Experiences and Relationships}

\section{Student researcher: Laura Duke, Senior}

What makes study abroad programs impactful? How does a participant's background and life journey affect their study abroad experience? Study abroad programs have become increasingly popular and more accessible across the country, especially at Purdue University. The Maymester in Tanzania study abroad program includes coursework and teaching in rural community schools. In addition, participants engage in service-learning projects and excursions. Through an investigation of participants' personal and professional life stories, we identified connections between the study abroad program, relationships, and impacts on the program and participants' lives. We developed a survey with multiple choice, Likert scale, and open-ended items to explore study abroad participants' contextual, curricular, and cocurricular experiences. The survey was sent to the 149 students who participated in the program between 2010 and 2018; 56\% of the participants responded. We used descriptive statistics to analyze the multiple choice and Likert scale items. Two researchers individually analyzed each open-ended question to identify response themes. The most common words used in open-ended responses were "people" and "students," which made us consider the importance of relationships in study abroad programs.

One participant highlighted the importance of community relationships, sharing, "They taught

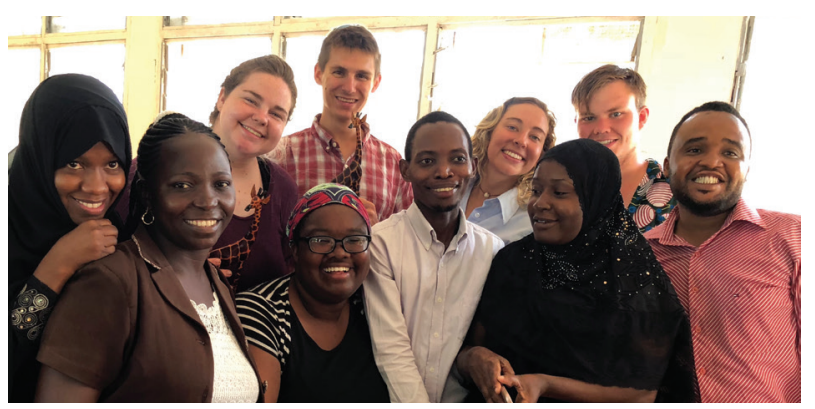

Laura Duke is pictured here with fellow Purdue students at Zingibari Secondary School in Tanzania with four teachers from the school. The students spent three weeks teaching every day alongside teachers from the school in various subject areas. During downtime at school, Purdue students learn Swahili and the Tanzanian culture from teachers.

us and we taught them. I think we impacted them through friendship and learning about different cultures just as much as they helped us.” Meaningful life experiences and knowledge production are intertwined with the formation and establishment of relationships. We hope to highlight the potential of this important and understudied aspect of study abroad experiences to enhance intercultural connections and learning.

Research advisor Jill Newton writes: "It has been a pleasure working with Laura on this study; she contributed important ideas to the design of the survey and analysis of the results. It is interesting that the importance of relationships emerged as a key finding given Laura's focus on developing crosscultural relationships as a program participant." 